\title{
STATISTICAL CAUSALITY AND QUASIMARTINGALES
}

\author{
DRAGANA VALJAREVIĆ ${ }^{1, \star}$, LJILJANA PETROVIĆ ${ }^{2}$ \\ ${ }^{1}$ Department of Mathematics, Faculty of Sciences and Mathematics, University of Priština, Kosovska Mitrovica, Serbia \\ ${ }^{2}$ Department of Mathematics and Statistics, Faculty of Economics, University of Belgrade, Belgrade, Serbia
}

\begin{abstract}
Concept of causality is very popular and applicable nowadays, especially when we consider the cases "what would happen if" and "what would have happened if". Here we consider the concept of causality based on the Granger's definition of causality, introduced in Mykland (1986). Many of the systems to which it is natural to apply tests of causality take place in continuous time, so we will consider the continuous time processes. Here we consider the connection between the concept of causality and the property of being a quasimartingale. Quasimartingales were investigated by Fisk (1965), Orey and specially Rao (1969). Namely, in this paper we prove an equivalence between the given concept of causality and preservation of quasimartingale property if the filtration is getting larger. We prove the same equivalence for the stopped quasimartingale with respect to the truncated filtrations.
\end{abstract}

Keywords: Causality, Filtration, Martingale, Quasimartingale.

\section{INTRODUCTION}

In this paper we consider a stochastic process $X_{t}$ which have a decomposition into the sum of a martingale process and a process having almost every sample function of bounded variation on the interval $I(I \subseteq \mathbb{R})$. Such a process is called a quasimartingale.

After Introduction, in Section 2 we give definition of the causality concept, based on the Granger's definition of causality and some basic properties of that concept which will be used later.

One of the goals of science is to find causal relations. This cannot always be done by experiments and researchers are restricted to observe the system they want to describe. This is the case in, e.g., economics, demography, etc. In the papers of Florens \& Mouchart (1982), Gill \& Petrovic (1987), Mykland (1986), Petrović (1996) it is shown how the conditional independence can serve as a basis for a general probabilistic theory of causality for both processes and single events.

The paper introduces a statistical concept of causality which unifies the nonlinear Granger-causality with some related concepts.

The linear Granger-causality was introduced by Granger, 1969. We shall study a nonlinear version of the concept. Like the linear one, it defines that the process $\mathbf{Y}=\left\{Y_{t}, t \in I\right\},(I \subseteq \mathbf{R})$ does not cause the process $\mathbf{X}=\left\{X_{t}, t \in I\right\}$ if, for all $t$, the orthogonal projection of the $L^{2}$-space representing $X_{s}, s>t$, on the space representing $X_{s}$ and $Y_{s}, s \leq t$ is contained in the space representing $X_{s}, s \leq t$. However, the spaces representing stochastic variables are those over the $\sigma$-field generated by these variables, while in the linear case they are the smallest closed linear spaces containing the variables.

We give a generalization of a causality relationship "G entirely causes $\mathbf{H}$ within $\mathbf{F}$ " which (in terms of $\sigma$-algebras) was introduced by Mykland (1986) and which is based on Granger's defini- tion of causality (see Granger (1969)) and discuss the relationship to nonlinear Granger-causality.

In Section 3, we consider relations between the given causality concept and the quasimartingale properties. More precisely, we analyze connection between causality and the preservation of the quasimartingale property with respect to the enlarged filtration $\mathbf{F}$ ( $\mathbf{F}$ is enlarged filtration of the natural filtration of quasimartingale $\mathbf{F}^{X}$ ).

The given concept of causality can be connected to the orthogonality of martingales (see Valjarević \& Petrović (2012)) and the stable subspaces of $H^{p}$ which contains the right continuous modifications of martingales (see Petrović \& Valjarević (2013)). The preservation of the predictable representation property, in the case when the information $\sigma$-algebra increases, is strongly connected to the concept of causality (see Petrović \& Valjarević (2014)). Also, the concept of statistical causality can be connected to the local weak solutions of stochastic differential equations driven with semimartingales (see Petrović \& Valjarević (2015)).

\section{NOTATIONS AND DEFINITIONS}

\section{Concept of causality}

Following Granger's and Sims's pioneering papers (see Sims (1972)), the notion of causality in econometric is generally defined within framework of prediction theory. This notion refers to situations in which it is possible to reduce the size of the information set that is taken into account for predicting a given variable $X_{1}$ without affecting the precision level of the prediction.

More precisely, a set of economic variables, denoted by $X_{2}$, does not cause a set of variables $X_{1}$, if the information available about $X_{2}$ may be forgotten without any consequence regarding the prediction of future $X_{1}^{\prime} s$. Since the content of the "available in- 
formation" set is not precisely described, the definition remains ambiguous.

Modern financial econometrics is mainly devoted to the study of rapidly evolving stochastic processes. The recent development of continuous time modelling in finance is an important motivation for considering the concept of causality in continuous time.

In this part of the paper we give the definition of the concept of causality relationship (in continuous time) between the flow of information (represented by filtrations) and between the stochastic processes.

Let $(\Omega, \mathcal{F}, P)$ be an arbitrary probability space and let $\mathbf{F}=$ $\left\{\mathcal{F}_{t}, t \in I(\subseteq \mathbf{R})\right\}$, be a family of sub- $\sigma$-algebras of $\mathcal{F} . \mathcal{F}_{t}$ can be interpreted as the set of events observed up to time $t$. Whether or not $\sup I=+\infty$ or $\inf I=-\infty$ we define $\mathcal{F}_{\infty}$ as the smallest $\sigma$ algebra containing all the $\mathcal{F}_{t}$ (even if $\sup I<+\infty$ ). So, we have $\mathcal{F}_{\infty}=\bigvee_{t \in I} \mathcal{F}_{t}$ and $\mathcal{F}_{-\infty}=\bigcap_{t \in I} \mathcal{F}_{t}$.

A filtration $\mathbf{F}=\left\{\mathcal{F}_{t}, t \in I\right\}$ is a nondecreasing family of sub- $\sigma$-algebras of $\mathcal{F}$, i.e. that is

$$
\mathcal{F}_{s} \subseteq \mathcal{F}_{t}, s \leq t
$$

A probabilistic model for a time-dependent system is described by $\left(\Omega, \mathcal{F}, \mathcal{F}_{t}, P\right)$, where $(\Omega, \mathcal{F}, P)$ is a probability space and $\left\{\mathcal{F}_{t}, t \in I\right\}$ is a "framework" filtration, i.e. $\left(\mathcal{F}_{t}\right)$ are all events in the model up to and including time $t$ and $\left(\mathcal{F}_{t}\right)$ is a sub- $\sigma$-algebra of $(\mathcal{F})$. We suppose that the filtration $\left(\mathcal{F}_{t}\right)$ satisfy the "usual conditions", which means that $\left(\mathcal{F}_{t}\right)$ is right continuous and each $\left(\mathcal{F}_{t}\right)$ is complete.

Analogous notation will be used for filtrations $\mathbf{H}=\left\{\mathcal{H}_{t}\right\}$ and $\mathbf{G}=\left\{\mathcal{G}_{t}\right\}, t \in I$.

It will be said that the filtrations $\mathbf{G}$ and $\mathbf{F}$ are equivalent (and written as $\mathbf{G}=\mathbf{F}$ ) if $\mathbf{G} \subseteq \mathbf{F}$ and $\mathbf{F} \subseteq \mathbf{G}$, or equivalently, if $\mathcal{G}_{t}=\mathcal{F}_{t}$ for each $t$.

A family of $\sigma$-algebras induced by a stochastic process $\mathbf{X}=$ $\left\{X_{t}, t \in I\right\}$ is given by $\mathbf{F}^{X}=\left\{\mathcal{F}_{t}^{X}, t \in I\right\}$, where

$$
\mathcal{F}_{t}^{X}=\sigma\left\{X_{u}, u \in I, u \leq t\right\},
$$

being the smallest $\sigma$-algebra with respect to which the random variables $X_{u}, u \leq t$ are measurable. The process $X_{t}$ is $\left(\mathcal{F}_{t}\right)$ adapted (or adapted to the filtration $\mathbf{F}=\left\{\mathcal{F}_{\mathbf{t}}\right\}$ ) if all $X_{u}, u \leq t$ are F-measurable, i.e. if

$$
\mathcal{F}_{t}^{X} \subseteq \mathcal{F}_{t} \text { for each } t
$$

The notation $\left(X_{t}, \mathcal{F}_{t}\right)$ means that $X_{t}$ is $\left(\mathcal{F}_{t}\right)$-adapted.

A family of $\sigma$-algebras may be induced by several processes, e.g. $\mathbf{F}^{X, Y}=\left\{\mathcal{F}_{t}^{X, Y}, t \in I\right\}$, where

$$
\mathcal{F}_{t}^{X, Y}=\mathcal{F}_{t}^{X} \bigvee \mathcal{F}_{t}^{Y}, t \in I .
$$

On the probability space $(\Omega, \mathcal{F}, P)$ the process $\mathbf{Z}=\left\{Z_{t}, t \in I\right\}$ is a $\left(\mathcal{F}_{t}, P\right)$-martingale if $Z_{t}$ is $\left(\mathcal{F}_{t}\right)$-adapted and $E\left(Z_{t} \mid \mathcal{F}_{s}\right)=Z_{s}$ for all $t \geq s$.
The intuitively plausible notion of causality formulated in terms of Hilbert spaces, is given in Petrović (1996). We shall use analogue notion of causality in terms of filtrations. Let $\mathbf{F}, \mathbf{G}$ and $\mathbf{H}$ be arbitrary filtrations. We can say that " $\mathbf{G}$ entirely causes $\mathbf{H}$ within $\mathbf{F} "$ if

$$
\mathcal{H}_{\infty} \perp \mathcal{F}_{t} \mid \mathcal{G}_{t}
$$

because the essence of $(1)$ is that $\left(\mathcal{G}_{t}\right)$ contains all information from the $\left(\mathcal{F}_{t}\right)$ needed for predicting $\mathcal{H}_{\infty}$. Let us mention that the condition $\mathbf{G} \subseteq \mathbf{F}$ does not represent essential restriction. Thus, it is natural to introduce the following definition of causality between filtrations.

Definition 1. (see Petrović (1996)) It is said that $\mathbf{G}$ entirely causes (or just causes) $\mathbf{H}$ within $\mathbf{F}$ relative to $P$ (and written as $\mathbf{H} \mid<$ $\mathbf{G} ; \mathbf{F} ; P)$ if $\mathcal{H}_{\infty} \subseteq \mathcal{F}_{\infty}, \mathbf{G} \subseteq \mathbf{F}$ and if $\mathcal{H}_{\infty}$ is conditionally independent of $\left(\mathcal{F}_{t}\right)$ given $\left(\mathcal{G}_{t}\right)$ for each $t$, i.e.

$$
\mathcal{H}_{\infty} \perp \mathcal{F}_{t} \mid \mathcal{G}_{t} \text { for each } t,
$$

(i.e. $\mathcal{H}_{u} \perp \mathcal{F}_{t} \mid \mathcal{G}_{t}$ holds for each $t$ and each $u$ ), or

$$
\left(\forall A \in \mathcal{H}_{\infty}\right) P\left(A \mid \mathcal{F}_{t}\right)=P\left(A \mid \mathcal{G}_{t}\right)
$$

If there is no doubt about $P$, we omit "relative to $P$ ".

The continuous time framework is fruitful, not only for the internal consistency of economic theories but also for the statistical approach to causality analysis between stochastic processes.

Intuitively, $\mathbf{H} K \mathbf{G} ; \mathbf{F}$ means that, for arbitrary $t$, information about $\mathcal{H}_{\infty}$ provided by $\left(\mathcal{F}_{t}\right)$ is not "bigger" than that provided by $\left(\mathcal{G}_{t}\right)$ or that it is possible to reduce available information from $\left(\mathcal{F}_{t}\right)$ to $\left(\mathcal{G}_{t}\right)$ in order to predict $\mathcal{H}_{\infty}$.

If $\mathbf{G}$ and $\mathbf{F}$ are such that $\mathbf{G} \mid<\mathbf{G} ; \mathbf{F}$, we shall say that $\mathbf{G}$ is its own cause within $\mathbf{F}$ (compare with Mykland (1986)). It should be mentioned that the notion of subordination (as introduced in Rozanov (1974)) is equivalent to the notion of being one's own cause, as defined here. It should be noted that "G is its own cause" sometimes occurs as a useful assumption in the theory of martingales and stochastic integration (see Bremaud \& Yor (1978), Revuz \& Yor (2005)).

These definitions can be applied to stochastic processes if we are talking about the corresponding induced filtrations. For example, $\left(\mathcal{F}_{t}\right)$-adapted stochastic process $X_{t}$ is its own cause if $\left(\mathcal{F}_{t}^{X}\right)$ is its own cause within $\left(\mathcal{F}_{t}\right)$, i.e. if

$$
\mathbf{F}^{X}<\mathbf{F}^{X} ; \mathbf{F} ; P \text {, holds. }
$$

Extensions of the definitions to vector processes are usually straightforward.

The process $X$ which is its own cause is completely described by its behavior relative to its natural filtration $\mathbf{F}^{X}$. For example, process $X=\left\{X_{t}, t \in I\right\}$ is a Markov process relative to the filtration $\mathbf{F}=\left\{\mathcal{F}_{t}, t \in I\right\}$ on a filtered probability space $\left(\Omega, \mathcal{F}, \mathcal{F}_{t}, P\right)$ if and only if $X$ is a Markov process relative to $\mathbf{F}^{X}$ and it is its own cause within $\mathbf{F}$ relative to $P$. 
The concepts of causality in continuous time are truly relevant for economic reasons (see Comte \& Renault (1996)).

In many situations we observe some system up to some random time, for example till the time when something happens for the first time. Definition 1 is extended from fixed times to stopping times in Petrović \& Valjarević (2016).

The $\sigma$-field $\left(\mathcal{F}_{T}\right)=\left\{A \in \mathcal{F}: A \cap\{T \leq t\} \in \mathcal{F}_{t}\right\}$ is usually interpreted as the set of events that occurs before or at time $T$ (see Elliot (1982)). For a process $X$, we set $X_{T}(\omega)=X_{T(\omega)}(\omega)$, whenever $T(\omega)<+\infty$. We define the stopped process $X^{T}=\left\{X_{t \wedge T}, t \in I\right\}$ with

$$
X_{t}^{T}(\omega)=X_{t \wedge T(\omega)}(\omega)=X_{t} \chi_{\{t<T\}}+X_{T} \chi_{\{t \geq T\}} .
$$

Note that if $X$ is adapted and cadlag and if $T$ is a stopping time, then the stopped process $X^{T}$ is also adapted.

Let us mention that the truncated filtration $\left(\mathcal{F}_{t \wedge T}\right)$ is defined as

$$
\mathcal{F}_{t \wedge T}=\mathcal{F}_{t} \cap \mathcal{F}_{T}=\left\{\begin{array}{l}
\mathcal{F}_{t}, t<T, \\
\mathcal{F}_{T}, t \geq T .
\end{array}\right.
$$

A martingale stopped at a stopping time is still a martingale. The natural filtration for the stopped martingale $X_{t \wedge T}$ is $\mathbf{F}^{X^{T}}=\left(\mathcal{F}_{t \wedge T}^{X}\right)$, with respect to which the process $X_{t \wedge T}$ is completely described. So, we have the definition of causality which involves the stopping times.

Definition 2. (Petrović \& Valjarević (2016)) Let $\mathbf{H}=\left\{\mathcal{H}_{t}\right\}$, $\mathbf{G}=\left\{\mathcal{G}_{t}\right\}$ and $\mathbf{E}=\left\{\mathcal{E}_{t}\right\}, t \in I$, be given filtrations on the probability space $(\Omega, \mathcal{F}, P)$ and let $T$ be a stopping time with respect to filtration $\mathbf{E}$. The filtration $\mathbf{G}^{T}$ entirely causes $\mathbf{E}^{T}$ within $\mathbf{H}^{T}$ relative to $P$ (and written as $\mathbf{E}^{T} K \mathbf{G}^{T} ; \mathbf{H}^{T} ; P$ ) if $\mathbf{E}^{T} \subseteq \mathbf{H}^{T}, \mathbf{G}^{T} \subseteq \mathbf{H}^{T}$ and if $\mathcal{E}_{T}$ is conditionally independent of $\mathcal{H}_{t \wedge T}$ given $\mathcal{G}_{t \wedge T}$ for each $t$, i.e. $(\forall t) \quad \mathcal{E}_{T} \perp \mathcal{F}_{t \wedge \tau} \mid \mathcal{G}_{t \wedge \tau}$, or

$$
(\forall t \in I)\left(\forall A \in \mathcal{E}_{T}\right) \quad P\left(A \mid \mathcal{H}_{t \wedge T}\right)=P\left(A \mid \mathcal{G}_{t \wedge T}\right) .
$$

The concept of causality given in Definition 2 includes the stopped filtrations. Namely, the causality relationship is defined up to a specified stopping time $T$.

\section{Quasimartingales}

The term quasimartingale is for the first time used by Fisk (1965). It is obvious that the sum and difference of two quasimartingales are again quasimartingales. The difference of two positive local martingales is necessarily a quasimartingale. Let us mention that there are some similarities between quasimartingales and supermartingales. Note that every finite set of random variables with expectations is trivially a quasimartingale. A mean right continuous quasimartingale always has a cadlag (right continuous with left limits) modification. Henceforth we will assume, unless otherwise stated, that all processes considered are cadlag at every time point.

Definition 3. (Protter, 2004) A finite tuple of points $\tau=$ $\left(t_{0}, t_{1}, \ldots, t_{n+1}\right)$ such that $0=t_{0}<t_{1}<\cdots<t_{n+1}=\infty$ is a partition of $[0, \infty]$.
Definition 4. (Protter, 2004) Suppose that $\tau$ is a partition of $[0, \infty]$ and that $X_{t_{i}} \in L^{1}$, each $t_{i} \in \tau$. Define

$$
C(X, \tau)=\sum_{i=0}^{n}\left|E\left(X_{t_{i}}-X_{t_{i+1}} \mid \mathcal{F}_{t_{i}}\right)\right| .
$$

The variation of $X$ along $\tau$ is defined to be

$$
\operatorname{Var}_{\tau}(X)=E(C(X, \tau)) .
$$

The variation of $X$ is defined to be

$$
\operatorname{Var}(X)=\sup _{\tau} \operatorname{Var}_{\tau}(X)
$$

where supremum is taken over all such partitions.

Definition 5. (Protter, 2004) An adapted, cadlag process $X$ is a quasimartingale on $[0, \infty]$ if $E\left(\left|X_{t}\right|\right)<\infty$, for each $t$, and if $\operatorname{Var}(X)<\infty$.

Next Theorem defines a Doob decomposition of quasimartingale.

Theorem 6. (Rao, 1969) A right continuous process $X_{t}$ is a quasimartigale if and only if it has a generalised Doob decomposition

$$
X_{t}=Y_{t}+M_{t}-B_{t}
$$

where $Y_{t}$ is a martingale, $M_{t}$ is the difference of two non-negative local martingales, and $B_{t}$ is the difference of two natural integrable increasing processes. This decomposition is unique.

The definition of natural integrable increasing process is given in Rao (1969).

\section{CAUSALITY AND QUASIMARTINGALES}

The certain results, not obvious from the definition of a quasimartingale or the fact that it is the difference of two supermartingales, follow from the decomposition from Theorem 6 . The starting point in this section is the decomposition

$$
X_{t}=M_{t}-B_{t}
$$

of a quasimartingale $X_{t}$ into a local martingale $M_{t}$ and a natural process with finite expected total variation $B_{t}$. This decomposition is unique.

Let $\left(\mathcal{G}_{t}\right)$ be a subfiltration of the filtration $\left(\mathcal{F}_{t}\right)$, i.e. $\left(\mathcal{G}_{t}\right) \subseteq$ $\left(\mathcal{F}_{t}\right)$. The next theorem holds.

Theorem 7. Every quasimartingale $X_{t}$ with respect to $\left(\mathcal{G}_{t}\right)$ is a quasimartingale with respect to $\left(\mathcal{F}_{t}\right)$ if and only if $\mathbf{G}$ is its own cause within $\mathbf{F}$, or equivalently if

$$
\mathbf{G K} \mathbf{G} ; \mathbf{F} ; P \text { holds. }
$$

Proof. Let the process $X_{t}$ be a $\left(\mathcal{G}_{t}\right)$ and $\left(\mathcal{F}_{t}\right)$ quasimartingale. From its unique decomposition (4) it follows that process $M_{t}$ is a $\left(\mathcal{G}_{t}\right)$ and $\left(\mathcal{F}_{t}\right)$-local martingale. According to Theorem 3.3 in Valjarević (2012), the causality $\mathbf{G} K \mathbf{G} ; \mathbf{F} ; P$ holds. 
Conversely, let $\mathbf{G}<\mathbf{G} ; \mathbf{F} ; P$ holds and let the process $X_{t}$ be a $\left(\mathcal{G}_{t}\right)$-quasimartingale. Then, the process $X_{t}$ has a unique decomposition $X_{t}=M_{t}-B_{t}$, where $M_{t}$ is a $\left(\mathcal{G}_{t}\right)$-local martingale. From $\mathbf{G K} \mathbf{G} ; \mathbf{F} ; P$ and Theorem 3.3 in Valjarević (2012) it follows that the process $M_{t}$ is $\left(\mathcal{F}_{t}\right)$-local martingale, too. Also, the process $B_{t}$ is a natural process with finite expected total variation with respect to filtration $\left(\mathcal{F}_{t}\right)$, because $\left(\mathcal{G}_{t}\right) \subset\left(\mathcal{F}_{t}\right)$. Hence, the process $X_{t}$ has a unique decomposition $X_{t}=M_{t}-B_{t}$ with respect to filtration $\left(\mathcal{F}_{t}\right)$, so it is a $\left(\mathcal{F}_{t}\right)$-quasimartingale.

Let $\mathbf{F}^{X}$ be a natural filtration of the quasimartingale $X_{t}$. Then the following theorem holds.

Theorem 8. Process $X_{t}$ is a $\left(\mathcal{F}_{t}\right)$-quasimartingale if and only if it is its own cause within $\left(\mathcal{F}_{t}\right)$, or equivalently if holds

$$
\mathbf{F}^{X} \in \mathbf{F}^{X} ; \mathbf{F} ; P \text {. }
$$

Proof. Follows directly by Theorem 7 (we set $\mathbf{G}=\mathbf{F}^{X}$ ).

Theorem 9. Let the process $X$ be uniformly integrable quasimartingale with respect to $\mathbf{G}$, let $T$ be a $\left(\mathcal{G}_{t}\right)$-stopping time and $\mathbf{G} \subset \mathbf{F}$. Then the stopped process $X^{T}=X_{t \wedge T}$ is quasimartingale with respect to $\mathbf{F}^{T}=\left\{\mathcal{F}_{t \wedge T}\right\}$ if and only if $\mathbf{G}^{T}$ is its own cause within $\mathbf{F}^{T}$, i.e. if

$$
\mathbf{G}^{T} K \mathbf{G}^{T} ; \mathbf{F}^{T} ; P \text { holds } .
$$

Proof. Let the process $X$ be uniformly integrable quasimartingale with respect to $\mathbf{G}, T$ be a $\left(\mathcal{G}_{t}\right)$-stopping time and

$$
\mathbf{G}^{T}<\mathbf{G}^{T} ; \mathbf{F}^{T} ; P \text {. }
$$

Due to Lemma I.1.8.12 in Skorohod \& Gikhman (2005) we have that $X_{T}$ is quasimartingale with respect to $\mathcal{G}_{T}$. According to the relation (5), from assumption of the theorem it follows that $X^{T}$ is quasimartingale with respect to $\mathbf{G}^{T}=\left\{\mathcal{G}_{t \wedge T}\right\}$. By Definition 5, Theorem 6 and assumption on the beginning of the Section it follows that the process $X^{T}$ can be represented as

$$
X^{T}=M^{T}-B^{T} .
$$

This decomposition is unique. Process $M^{T}$ is martingale with respect to $\mathbf{G}^{T}$. According to Theorem 6 in Petrović \& Valjarević (2016), from (5) it follows that the process $M^{T}$ is martingale with respect to $\mathbf{F}^{T}$, too. Using the same technique as in the previous proof, we get that $B^{T}$ is a process of bounded variation with respect to $\mathbf{G}^{T}$ and $\mathbf{F}^{T}$, too $\left(\mathbf{G}^{T} \subset \mathbf{F}^{T}\right)$. So, process $X^{T}$ can be presented as $X^{T}=M^{T}-B^{T}$ with respect to $\mathbf{F}^{T}$, where $M^{T}$ is a local martingale and $B^{T}$ is a process of bounded variation.

Conversely, suppose that $X^{T}$ is quasimartingale with respect to $\mathbf{G}^{T}$ and $\mathbf{F}^{T}$, where $T$ is a $\left(\mathcal{G}_{t}\right)$-stopping time. Due to decomposition of the quasimartingale and its uniqueness, follows that $X^{T} M^{T}-B^{T}$ is unique decomposition with

$\mathbf{F}^{T}$. So, $M^{T}$ is martingale with respect to filtrations $\mathbf{G}^{T}$ and $\mathbf{F}^{T}$.

Due to Theorem 6 in Petrović \& Valjarević (2016), it follows that Skorohod, I. \& Gikhman, L. 2005. Stochastic processes. New York: $\mathbf{G}^{T} K \mathbf{G}^{T} ; \mathbf{F}^{T} ; P$ holds.

\section{REFERENCES}

Bremaud, P. \& Yor, M. 1978. Changes of filtrations and of probability measures. Zeitschrift fur Wahrscheinlichkeitstheorie und Verwandte Gebiete, 45(4), pp. 269-295. doi:10.1007/bf00537538.

Comte, F. \& Renault, E. 1996. Noncausality in Continuous Time Models. Econometric Theory, 12(02). doi:10.1017/s0266466600006575.

Elliot, R. J. 1982. Stochastic Calculus and applications.New York: Springer-Verlag.

Fisk, D. L. 1965. Quasi-Martingales. Transactions of the American Mathematical Society, 120(3). doi:10.2307/1994531.

Florens, J. P. \& Mouchart, M. 1982. Note on Noncausality. Econometrica, 50(3). doi:10.2307/1912602.

Gill, J. B. \& Petrovic, L. 1987. Causality and Stochastic Dynamic Systems. SIAM Journal on Applied Mathematics, 47(6), pp. 1361-1366. doi:10.1137/0147089.

Granger, C. W. J. 1969. . Investigating Causal Relations by Econometric Models and Cross-spectral Methods. Econometrica, 37(3). doi:10.2307/1912791.

Mykland, P. A. 1986. Statistical Causality. Report, 2, pp. 1-21.

Petrović, L. 1996. Causality and Markovian representations. Statistics Probability Letters, 29(3), pp. 223-227. doi:10.1016/0167-7152(95)00176-x.

Petrović, L. \& Valjarević, D. 2013. Statistical causality and stable subspaces of the australian mathematical society. Bulletin of the Australian Mathematical Society, 88(01), pp. 17-25. doi:10.1017/s0004972712000482.

Petrović, L. \& Valjarević, D. 2014. Statistical causality and martingale representation property with application to stochastic differential equations. Bulletin of the Australian Mathematical Society, 90(02), pp. 327-338. doi:10.1017/s000497271400029x.

Petrović, L. \& Valjarević, D. 2015. Lecture Notes in Computer Science: Statistical Causality and Local Solutions of the Stochastic Differential Equations Driven with Semimartingales.Cham: Springer Nature America, Inc., pp. 261-269. doi:10.1007/978-3-319-15765-8,4.

Petrović, L.and Dimitrijević, S. \& Valjarević, D. 2016. Granger causality and stopping times*. Lithuanian Mathematical Journal, 56(3), pp. 410-416. doi:10.1007/s10986-016-9325-0.

Protter, P. 2004. Stochastic Integration and Differential Equations.Berlin: Springer-Verlag.

Rao, K. M. 1969. Quasi-Martingales. Mathematica scandinavica, 24. doi:10.7146/math.scand.a-10921.

Revuz, D. \& Yor, M. 2005. Continuous martingales and Brownian motion. New York: Springer.

Rozanov, Y. A. 1974. Theory of Innovation Processes. Monographs in Probabolity Theory and Mathematical Statistics.Moscow: Izdat Nauka.

Sims, C. A. 1972. Money, income and causality. American Economic Review, 62, pp. 540-552.

Springer. 1 
Valjarević, D. 2012. Theory of statistical causality, stochastic differ- Valjarević, D. \& Petrović, L. 2012. Statistical causality and orthogential equations and martingale representation property. Univer- onality of local martingales. Statistics Probability Letters, 82(7), sity thought, 82, pp. 1326-1330. pp. 1326-1330. doi:10.1016/j.spl.2012.03.036. 